selves, both in the embryonic epidermis and in the adult central nervous system (CNS).

These results suggest that the earliest signs of morphological segmentation (for example, somite formation, in the case of vertebrates) may be very poor indicators of the basic mechanisms which generate periodicity, and that it would be unsafe to rely on such criteria to assess the phylogenetic origin of segmentation. Indeed, if the importance of periodic organization is recognized, then the question whether segmentation did or did not arise independently in different phyla becomes irrelevant.

An alternative approach to the question would be to look for the earliest signs of periodic organization in metazoans. Architecture of the CNS across the major phyla shows that periodic organization of the CNS is a common feature of most of them, including some of the most primitive flatworms ${ }^{3}$. Furthermore, periodicity is obvious in the CNS of primitive molluscs (chiton) and in echinoderms (starfish), even though overt body segmentation is lacking or doubtful in these organisms.

This suggests that the principle of periodicity (or metamerization) may have emerged in the CNS before it was applied to any other tissue, possibly to help set up a scaffold of increasingly complex stereotyped connections. Indeed, basic determinants of the CNS structure, such as the paths laid down by pioneer neurons, have evolved remarkably little over most of the arthropod lineage ${ }^{4}$, presumably because of the structural constraints involved in the establishment of a stereotyped pattern of connections.

In this view, periodic organization would definitely have arisen (in the CNS) before the separation of the major lineages. Recent work on the segmentation of the hindbrain in vertebrates and its correlation to the realms of action of $H o x$ genes $^{6}$ are certainly consistent with this view.

Université Libre de Bruxelles,

Laboratoire de Genetique,

Rue des Chevaux 67,

1640 Rhode-Saint-Genèse, Belgium

1. Wilkinson, D.G. et al. Nature 341, 405-409 (1989)

2. Ingham, P. Nature $335,25-34$ (1989).
3. Bullock, T.H. \& Horridge, G.A. Structure and function in the nervous system of invertebrates (Freeman, San Francisco, 1965).

4. Thomas, J.B. et al. Nature 310, 203-207 (1984).

5. Lumsden, A. \& Keynes, R. Nature 337, 424-428 (1989)

6. Murphy, P. et al. Nature 341, 156-159 (1989)

\section{Scientific Correspondence}

Scientific Correspondence is intended to provide a forum in which readers may raise points of a scientific character. They need not arise out of anything published in Nature. In any case, priority will be given to letters of fewer than 500 words and five references.

\title{
Genetic linkage and psychiatric disease
}

SIR-The claim by Robertson in her News and Views article ${ }^{1}$ that there is no persuasive evidence linking any psychiatric disease to a single locus is incorrect. There is a well-established linkage for manic depression on the $\mathrm{X}$ chromosome, which was first reported 20 years $\mathrm{ago}^{2}$ and has since been confirmed in Sardinia, Belgium, Israel and the United States in independent samples ${ }^{3-7}$. In the Israeli sample the lod score was greater than 9.00 , which is highly significant. When all the studies showing $X$ linkages are combined, the lod is greater than 15 .

Robertson also suggested that the chromosome 5 linkage for schizophrenia reported from our laboratory was incorrect: her comment was based on a misleading interpretation of our unpublished data by Ken Kidd at Nature's recent conference on molecular genetics and Human Disease. The marker M4, which lies inbetween the two markers that we have already published as being linked to schizophrenia, does in fact show linkage to schizophrenia with a lod of 1.53 . The reason that it is not higher than this is because it is not providing as much information from the seven families as the other two markers.

This is one of the difficulties commonly encountered in linkage research because it cannot be guaranteed that key individuals included in an analysis will be heterozygous for the marker polymorphisms and thus become informative for linkage. Unfortunately this is due to chance. In our families, M4 is occasionally informative in the same individuals as the marker p105$599 \mathrm{Ha}$, which shows a two-point lod to schizophrenia of 4.95 for the broadest definition of affection. Where it is informative, M4 gives exactly the same linkage information as p105-599Ha, except that in one family (F20) two individuals who were previously uninformative now become cases of incomplete penetrance. We originally argued ${ }^{8}$ that the presence of incomplete penetrance for schizophrenia would make it difficult to estimate accurately the recombination fraction between linkage markers and the chromosome 5 schizophrenia locus, unless our 'penetrance-free' model was used.

Robertson also states that readers of Nature should be sceptical of linkages to psychiatric disorders that have not reached a lod of 6 . There is no reason why

. Robertson, M. Nature 342, 222 (1989)

2. Winokur, G., Clayton, P.J. \& Reich, T. Manic Depressive IIIness (Mosby, St Louis, 1969).

3. Mendlewicz, J., Fleiss, J.L. \& Gieve, R.R. J. Am. med. Assn 222, 1624-1627 (1972)

4. Del Zompo, M. et al. Acta Psychiat. Scand. 70, 828-837 (1984).

5. Risch, N. \& Baron, M. Ann. hum. Genet. 46, 153-166 (1982).

6. Baron, M. et al. Nature 326, 289-292 (1987)

7. Mendlewicz, J. et al. Lancet I, 1230-1231 (1987).

8. Sherrington, R. et al. Nature 336, 164-167 (1988). psychiatric disorders should be singled out for special treatment, except for the fact that some psychiatric disorders show incomplete penetrance, and that the penetrance must be estimated jointly with the recombination fraction. If this is done, then the appropriate level of significance is a lod of 3.85. The presence of heterogeneity of linkage, uncertainty about definitions of caseness, and the presence of phenocopies very much reduces the chance of spurious linkage being reported for psychiatric disorders and this should alert us to the possibility that false negative linkages are more likely to occur than spuriously positive ones.

HUGH GURLING

Molecular Psychiatry Laboratory,

University College and Middlesex School of Medicine,

University of London,

W1P 7PN UK

SIR - Miranda Robertson's lucid discussion $^{1}$ of the problems already generated by the application of linkage methods appropriate to mendelian disorders to disorders that rarely show evidence of mendelian segregation overlooked two of the central quicksands on which the foundation of this claim rested, as indeed did your referees of the paper of Egeland et al. in 1987 (ref. 2).

First, in the analysis of a single marker, it is not sufficient to consider only the prior odds against the occupancy of a locus by a particular allele, which in man is of the order of twice the number of chromosomes, so that odds of a thousand-to-one, even if calculated after defining the maximum, is likely to be in error in only about 5 per cent of apparent linkages. It is also necessary to consider the prior odds against such a simple mechanism: the prior expectation that a functional disorder with very variable manifestation in an organ of extreme complexity of structure and function will have a simple mechanism must have some consideration, even if highly subjective. The possibility of two or more strongly predisposing alleles at different loci is at least more likely than one, but as there are more than a thousand ways in which two loci can be chosen with fairly distinct segregation, this approach is also limited.

The Amish study was complicated by a more serious problem, one underlying the optimistic predictions of the scope of linkage in the original article of Botstein et al. ${ }^{3}$ that spawned the term "the new genetics": the more marker loci are tested, the more likely the discovery of a false linkage due to fortuitous segregation. The Amish study of 1987 was based on a previous trawl of the genome with twenty markers. When one of these was found to cosegregate, a set of markers known to be closely 